\title{
¿Está disminuyendo la población de gaviota patiamarilla cantábrica Larus michahellis lusitanius Naumann, 1840? Censo 2013/2014 de Bizkaia (País Vasco) \\ Is the population of Cantabrian yellow-legged gull Larus michahellis lusitanius Naumann, 1840 decreasing? 2013/2014 Census in Biscay (Basque Country)
}

Aitor Galarza ${ }^{1 *}$

*

\section{Resumen}

En los años 2013 y 2014 se censó el número de parejas reproductoras de gaviota patiamarilla cantábrica Larus michahellis lusitanius Naumann, 1840 en la costa de Bizkaia (País Vasco). Los resultados se compararon con los del censo anterior, llevado a cabo en 2007. Se apreció una reducción global del 46,7\% (pasando de 3.702 a 1.973, una reducción de 1.740 parejas reproductoras). Cinco de las seis colonias con mayor número de parejas reproductoras sufrieron disminuciones cercanas o superiores al 50\%. Se baraja como causa probable de la reducción la disminución de recursos, esencialmente como consecuencia del cierre de vertederos de residuos orgánicos, y se alerta sobre las consecuencias que puede tener sobre la población total de gaviota patiamarilla cantábrica.

Palabras clave: vertederos, disponibilidad trófica, población, mortalidad de adulto.

\section{Abstract}

During 2013 and 2014, the Cantabrian yellow-legged gulls Larus michahellis lusitanius Naumann, 1840 breeding in the coast of Biscay were counted. Results were compared with the

\footnotetext{
1 Diputación Foral de Bizkaia. Departamento de Agricultura

E-48014 • Bilbao

* Correspondencia: aitorgalarzai@gmail.com
} 
previous census, conducted in 2007. An overall reduction of $46.7 \%$ was observed (from 3,702 to 1,973 , a reduction of 1,740 breeding pairs). Five of the six colonies with the highest numbers of breeding pairs suffered near or above a $50 \%$ of decrease. It is suggested that the probable cause of the population decrease is the decline of food resources, essentially following the closure of landfills, which might have negative consequences on Cantabrian yellow-legged gull at a broader geographic scale.

Key words: landfills, trophic availability, population, adult mortality.

\section{Laburpena}

2013 eta 2014 urteetan Bizkaiko kostaldeko Kantauriko kaio hankahoriaren Larus michahellis Iusitanius Naumann, 1840 bikote habiagileren kopurua zenbatu zen. 2007an eginiko aurreko zentsuko datuekin alderatuta, \%46,7ko osoko murrizketa hauteman zen (3.702tik 1.973ra: 1.740 bikote habiagile gutxiago). Bikote habiagile gehien duten sei kolonietatik bostek \%50 inguruko edo gehiagoko murrizketak izan zituzten. Murrizketa horren balizko kausa gisa hondakin-zabortegien itxierak sorturiko baliabide trofikoen gutxitzea iradokitzen da eta murrizketa horrek Kantauriko kaio hankahoriaren populazio osoan izan ditzakeen ondorioei buruz ohartarazten da.

Gako hitzak: zabortegiak, eskuragarrintasun trofikoa, populazioa, helduen hilkortasuna.

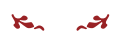

Las poblaciones de varias especies de aves marinas aumentaron de forma espectacular en Europa durante el siglo pasado como consecuencia de la protección de sus colonias de cría y sobre todo debido al incremento de la cantidad de alimento disponible, procedente principalmente de vertederos y descartes pesqueros (Pons \& Migot, 1995; Grémillet \& Boulinier, 2009). No obstante, estudios recientes indican que en las últimas décadas algunas de estas especies han visto reducidas sus poblaciones, esencialmente como respuesta a una caída en la disponibilidad trófica, consecuencia de una reducción de los descartes pesqueros y cambios en la política de tratamiento de los residuos urbanos (Oro et al., 2013; Newton, 2013). Así, por ejemplo, en el Reino Unido el número de parejas reproductoras de gaviota argéntea, Larus argentatus L. 1758, decreció un 36\% en el período 2000-2012 (INCC, 2013), lo que se suma a un declive del 57,2\% en los 30 años anteriores (Mitchell et al., 2006).

En la península Ibérica la especie de ave marina más beneficiada por el incremento de alimento procedente de vertederos y descartes pesqueros fue probablemente la gaviota patiamarilla, Larus michahellis, que únicamente en España mantenía una población de unas 125.000 parejas reproductoras a finales de los años noventa (Molina, 2009). Los ejemplares de esta especie que anidan en la costa atlántica de la Península, desde Portugal 
hasta el País Vasco, pertenecen a la subespecie lusitanius, siendo una población con un comportamiento marcadamente sedentario (Arizaga et al., 2010), frente al comportamiento en parte migratorio de la población mediterránea, perteneciente a la subespecie michahellis (Galarza et al., 2012). Esta población atlántica estaba compuesta en España por 63.274 parejas según el último censo, de las cuales alrededor del 7\% anidaba en el País Vasco (Molina, 2009). En el País Vasco la población de gaviota patiamarilla experimentó un incremento cercano al 150\% durante el período 1980-2000, lo que se ha relacionado con el aumento de la disponibilidad trófica (Arizaga et al., 2009). Al final de este período el incremento pareció ralentizarse, aunque algunas colonias continuaron creciendo en la década de 2000 (Arizaga et al., 2009). Más recientemente, el seguimiento de tres colonias en Gipuzkoa (País Vasco) apunta a un ligero descenso poblacional desde el año 2000, si bien éste no se consideró significativo (Arizaga et al., 2014).

La adaptación a la estrategia comunitaria (Directiva 1999/31/UE) de la política de tratamiento de residuos urbanos en Bizkaia (PIGRUB, 2012) ha supuesto la reducción en un 87,37\% de la cantidad de desechos depositados en este territorio en el periodo 1999-2014 (Fig. 1) y ha conllevado la clausura de varios vertederos desde finales de los años 1990 (Barakaldo, Gordexola, Getxo y Amoroto), y en 2013 el cierre de dos de los últimos, situados en Igorre y Lemoiz. Además, el vertedero de Bilbao, el único aún sin clausurar, redujo paulatinamente a partir de 2004 la cantidad de residuos disponibles al iniciar el tratamiento

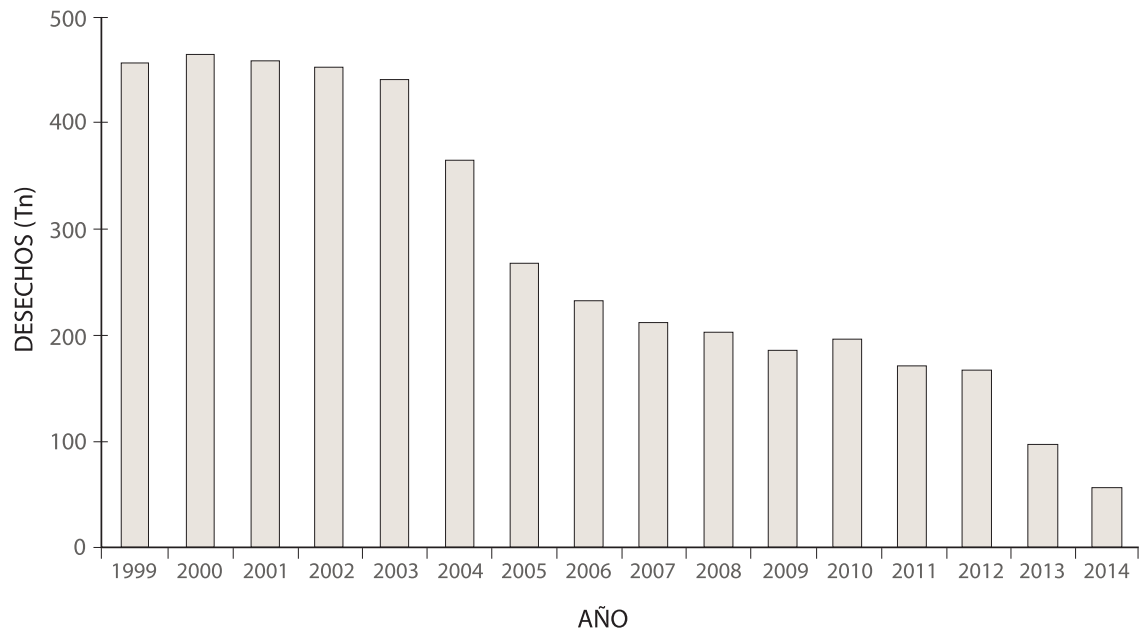

Fig. 1.- Evolución de la cantidad (Tn) de residuos depositados en los vertederos de Bizkaia en el periodo 1999-2014 (Fuente: Observatorio Permanente de Residuos Urbanos del Territorio Histórico de Bizkaia, www.bizkaia.net).

Fig. 1.- Amount of waste (Tn) dumped in landfills of Biscay in the period 1999-2014 (Source: Permanent Observatory of Urban Waste of Biscay). 


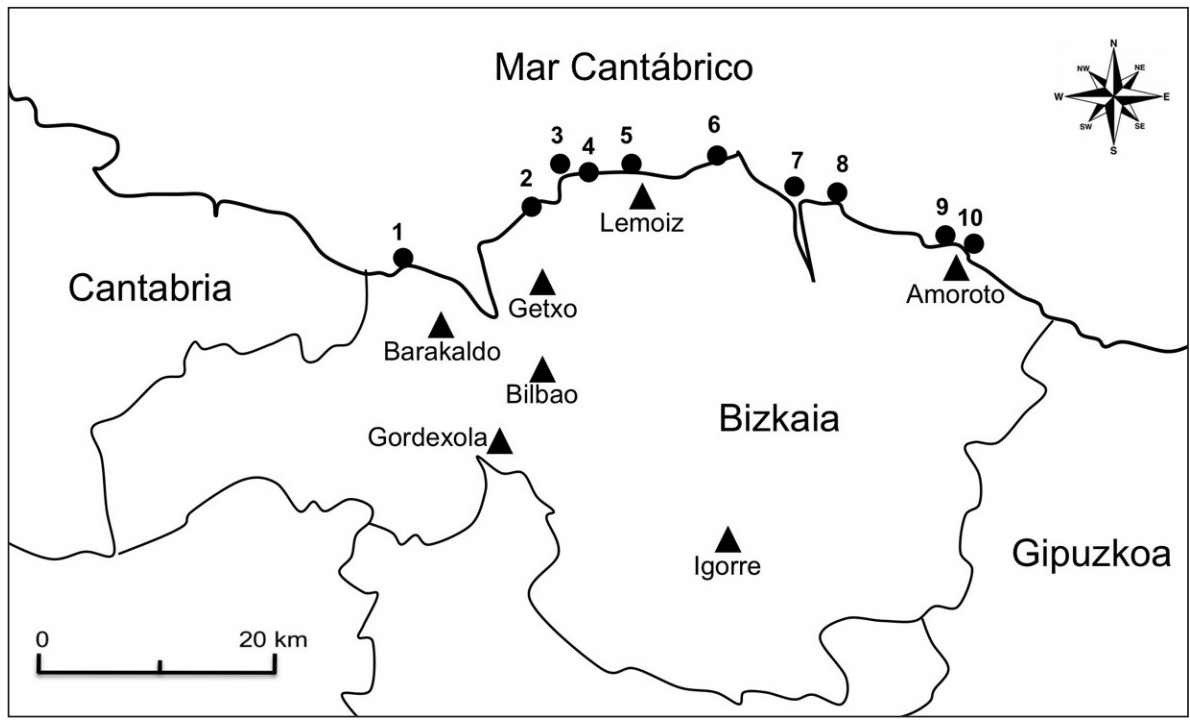

1 Punta Lucero, 2 Barrika, 3 Isla Billano, 4 Arminza, 5 Central nuclear (Lemoiz), 6 Isla Aketx, 7 Isla Izaro, 8 Ogoño, 9 Tala (Lekeitio), 10 San Nicolás (Lekeitio).

Fig. 2.- Localización de vertederos (triángulos) y principales colonias de gaviota patiamarilla (círculos) en Bizkaia.

Fig. 2.- Landfills (triangles) and main breeding colonies (circles) in Biscay.

por incineración y desarrolla desde hace años un programa de cetrería que dificulta eficazmente el acceso de las gaviotas a los residuos no tratados. Todos estos vertederos están localizados a menos de 30 km de distancia de la costa (Fig. 2) y, aunque no se dispone de datos de alimentación en la zona de estudio, el anillamiento desde 2004 y posterior lectura de ejemplares indica que tanto los adultos como los juveniles de gaviota patiamarilla de Bizkaia utilizaron asiduamente los desechos de la mayoría de estos vertederos durante sus períodos de actividad.

Durante el año 2013 se llevó a cabo el censo de la mayoría de las colonias de Bizkaia, censo que se completó en 2014, con el objetivo de determinar si se había producido un cambio significativo del número de parejas reproductoras desde el último censo, llevado a cabo en 2007. Los censos se realizaron durante la última quincena de mayo y la primera de junio mediante el conteo directo de los nidos y/o parejas, bien desde el exterior de la coIonia cuando era visible a distancia, o bien accediendo a ésta si la perspectiva o la distancia dificultaba o impedía el conteo (véase Molina, 2009). La diferencia de abundancia entre los dos censos fue testada mediante una prueba de la t para muestras pareadas. Los datos fueron transformados $\left(\log _{10}(x+1)\right)$, verificándose su normalidad con la prueba de Shapiro-Wilk. Los análisis se realizaron mediante el paquete estadístico IBM SPSS versión 22.

En la Tabla 1 se muestran los resultados del censo de parejas reproductoras de los dos censos citados, indicando el número de parejas por colonia, así como el total estimado 


\begin{tabular}{|lccc|}
\hline Colonia & 2007 & $2013 / 14$ & Dif. $\%$ \\
\hline Isla Izaro (Bermeo) & 1.273 & 795 & $-37,7$ \\
Isla San Nicolás (Lekeitio) & 881 & 451 & $-48,8$ \\
Tala (Lekeitio) & 439 & 188 & $-57,1$ \\
Isla Aketx (Bermeo) & 408 & 216 & $-47,1$ \\
Barrika & 226 & 58 & $-74,3$ \\
Ogoño (Elantxobe) & 181 & 11 & $-93,9$ \\
Punta Lucero (Zierbena) & 63 & 102 & $+61,7$ \\
Isla Billano & 48 & 28 & $-41,6$ \\
Arminza & 35 & 0 & -100 \\
Lemoiz (Central nuclear) & 28 & 52 & $+46,1$ \\
Bermeo (Casco urbano) & 26 & 29 & $+11,5$ \\
Ea/lbarrangelu & 20 & 10 & $-50,0$ \\
Islotes Bakio & 18 & 12 & $-33,3$ \\
Islote Kaiarria (Ea) & 17 & 6 & $-64,7$ \\
Laga (Ibarrangelu) & 17 & 0 & -100 \\
Otoio (Ispaster) & 9 & 13 & $+44,4$ \\
Larra (Ea) & 6 & 1 & $-83,3$ \\
Mundaka (Casco urbano) & 3 & 1 & $-66,6$ \\
Sestao (Casco urbano) & 3 & -973 & - \\
Muskiz (Casco urbano) & 1 & 29,7 \\
\hline TOTAL & 3.702 & 29 & - \\
\hline
\end{tabular}

Tabla 1.- Número de parejas reproductoras de gaviota patiamarilla en Bizkaia en 2007 (Arizaga et al., 2009) y 2013/14 y porcentaje de variación entre ambos censos (Dif\%).

Table 1.- Number of Yellow-legged gull breeding pairs in Biscay in 2007 (Arizaga et al., 2009) and 2013/14, and percentage difference between both census (Dif\%).

para Bizkaia. Se observó diferencia significativa entre las medias de ambos censos ( $t=2,620$, g.l.=17, p=0,018), apreciándose una reducción del número de parejas reproductoras $(46,7 \%)$ que pasaron de 3.702 a 1.973 (1.729 parejas menos). Ello refleja que la población de gaviota patiamarilla ha sufrido un importante y rápido declive en la zona de estudio durante el último sexenio. Cinco de las seis colonias con mayor número de parejas reproductoras sufrieron disminuciones significativas, cercanas o superiores al 50\% (San Nicolás, Tala, Aketx, Barrika y Ogoño), destacando la disminución del 94\% de la colonia de Ogoño. La disminución fue algo menos acusada $(37,7 \%)$ en la isla de Izaro, la mayor de las colonias, pero debe tenerse en cuenta que esta colonia ya había disminuido un 20\% en el periodo 2002-2007 (Arizaga et al., 2009). En dos de las colonias (Arminza y Laga) no se detectaron indicios de reproducción en el nuevo censo. Por el contrario, otras dos colonias (Punta Lu- 
cero y Lemoiz), ambas formadas en la década de 2000, destacaron por aumentar sus efectivos de forma importante (61,7 y 46,1\%, respectivamente). Tanto en las colonias del casco urbano de Mundaka como en la de Bermeo se llevaron a cabo descastes durante el periodo de estudio lo que no permite conocer cuál habría sido su evolución natural.

La reducción de efectivos en poblaciones de especies oportunistas como respuesta a una disminución en la disponibilidad de los recursos tróficos generados por el hombre es un fenómeno conocido tanto en gaviotas (Pons, 1992; Dunhem et al., 2008) como en otras aves (Olea \& Baglioni, 2008; Saurola, 2009). Esta reducción puede estar causada por la disminución del éxito reproductor, por el incremento de la mortalidad, por la emigración hacia zonas más favorables, o por una combinación de estos factores (Bosch et al., 2000, Newton, 2013).

El número de pollos anillados anualmente en la isla de Izaro se ha reducido en los últimos años (Fig. 3), lo que sugiere una menor productividad en esta colonia, la más importante del área de estudio. Además, la supervivencia de los ejemplares de primer año de esta misma colonia en los últimos años fue considerablemente menor a la que se observó en varias colonias cercanas, fuera ya del área de estudio (Juez et al., 2015). Aún así, una disminución del número de jóvenes producidos o un incremento de su mortalidad no explicaría por si sola la rápida reducción de la población observada en el transcurso de un sexenio en una especie cuya madurez sexual no tiene lugar hasta el cuarto año calendario. Datos preliminares derivados de la captura y recaptura de ejemplares adultos reproductores de la isla de Izaro (obs. pers.) descartan la emigración de adultos reproductores hacia colonias

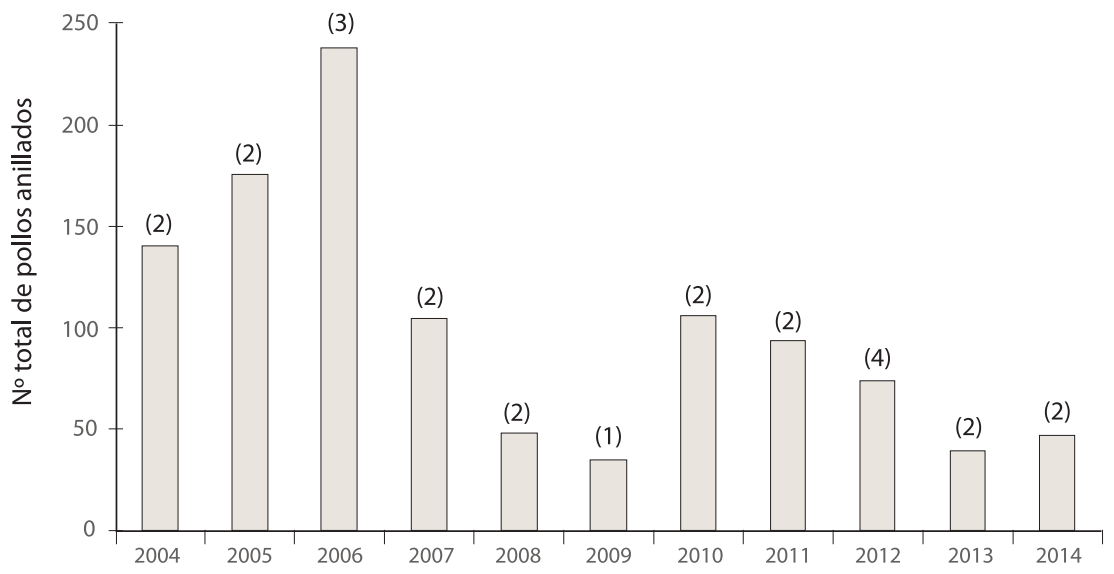

Fig. 3.- Número de pollos de gaviota patiamarilla anillados anualmente en la isla de Izaro (2004-2014). Se indica entre paréntesis el número anual de jornadas de anillamiento.

Fig. 3.- Number of Yellow-legged gull chicks annually ringed in Izaro Island (2004-2014). The number of ringing days per year is shown in brackets. 
situadas en otras zonas de la cornisa cantábrica y parecen apuntar a una elevada mortalidad de los reproductores como uno de los factores principales reguladores del tamaño de la población de gaviota patiamarilla en Bizkaia durante el último sexenio.

Dado que se han observado diferencias intercoloniales significativas en la alimentación de las gaviotas patiamarillas del País Vasco (Arizaga et al., 2013), cabe esperar diferencias intercoloniales en la magnitud y velocidad del impacto causada por la disminución de alimento procedente de los vertederos. Por ello, la tendencia opuesta a la general observada en dos de las colonias censadas podría estar relacionada con un menor uso de los vertederos por parte de los integrantes de estas colonias. No obstante, hay que tener en cuenta también que ambas son pequeñas colonias formadas hace pocos años y, por tanto, podrían estar menos sujetas a mecanismos denso-dependientes, lo que favorecería a corto plazo un mayor reclutamiento (Raven \& Coulson, 1997), atrasando en el tiempo el impacto derivado de la disminución de recursos.

Se desconoce el impacto global que el cierre de vertederos está teniendo en la población de gaviota patiamarilla que se reproduce o alimenta en la fachada atlántica de la península Ibérica. No obstante, teniendo en cuenta lo observado en Bizkaia en el último sexenio, resulta predecible que el paulatino cumplimiento de la Directiva del Consejo de Europa (1999/31/UE) traspuesta en el Real Decreto 1481/2001, que insta a la reducción de la cantidad de residuos biodegradables, vaya a afectar seriamente a toda la población de la subespecie lusitanius y, probablemente también a la fracción migradora michahellis, de presencia verano-otoñal en esta región (Galarza et al., 2012). Esta situación podría verse agravada por la normativa europea que promueve la reducción de descartes pesqueros (Regulación EU 1380/2013), otra de las fuentes importantes de alimentación de las gaviotas patiamarillas en el golfo de Bizkaia (Arizaga et al., 2013).

En este contexto, una especie que a menudo ha sido considerada como plaga (Oro \& Martínez-Abraín, 2007), podría entrar en un notable declive, al igual que ha ocurrido con la gaviota argéntea en Gran Bretaña (Mitchell et al., 2006; INCC 2013), recientemente incorporada a la lista roja de las aves británicas (Eaton et al., 2009). Por ello, como medida precautoria resultaría conveniente garantizar la protección y conservación de las colonias de cría, y eliminar los programas de descaste interviniendo únicamente sobre aquellas colonias urbanas en los que quede demostrado un importante perjuicio para la población humana, siempre bajo autorización de la administración competente y basando las actuaciones en el mejor conocimiento posible.

\section{Agradecimientos}

Pep Arcos, David Álvarez, Iván de la Hera y Pilar Rodriguez contribuyeron con sus comentarios a mejorar una primera versión del trabajo. Jon Hidalgo, Ignacio García, Gorka Ocio, Maider Olondo, Fran Martínez, Maite Martínez-Madrid, Pablo Pérez, Eneko Díaz y otros muchos voluntarios colaboraron en el anillamiento de ejemplares en la isla de Izaro. 


\section{Bibliografía}

- Arizaga, J., Galarza, A., Herrero, A., Hidalgo, J., Aldalur, A. 2009. Distribución y tamaño de la población de gaviota patiamarilla Larus michahellis lusitanius en el País Vasco, tres décadas de estudio. Revista Catalana d'Ornitologia 25: 32-42.

- Arizaga, J., Herrero, A. Galarza, A., Hidalgo, J., Aldalur, A., Cuadrado, J.F. Ocio, G. 2010. First-year movements of Yellow-legged gull (Larus michahellis lusitanius) from Southeastern Bay of Biscay. Waterbirds 33(4): 444-450.

- Arizaga, J., Jover, LI., Aldalur, A., Cuadrado, J.F., Herrero, A., Sanpera, C. 2013. Trophic ecology of a resident Yellow-legged Gull (Larus michahellis) population in the Bay of Biscay. Mar. Environ. Res. 87-88: 1-7.

- Arizaga, J., Aldalur, A., Herrero, A. 2014. Tendencia poblacional en tres colonias de gaviota patiamarilla Larus michahellis Naumann, 1840 en Gipuzkoa: 2000-2013. Munibe, Cienc. Nat. 62: 103-115.

- Bosch, M., Oro, D., Cantos, F.J., Zabala, M., 2000. Short-term effects of culling on the ecology and population dynamics of the Yellow-legged gull. J. Appl. Ecol. 37: 369-385.

- Dunhen, C., Roche, P., Vidal, E., Tatoni, T. 2008. Effects of anthropogenic resources on yellowlegged gull colony size on Mediterranean islands. Popul. Ecol. 50:91-100.

- Eaton, M.A., Brown, A.F., Noble, D.G., Musgrove, A.J., Hearn, R., Aebischer, N.J., Gibbons, D.W., Evans, A., Gregory, R.D. 2009. Birds of Conservation Concern 3: the population status of birds in the United Kingdom, Channel Islands and the Isle of Man. Br. Birds 102: 296-341.

- Galarza, A., Herrero, A., Domínguez, J.M., Aldalur, A., Arizaga, J. 2012. Movements of Mediterranean Yellow-legged gulls Larus michahellis to the Bay of Biscay. Ringing \& Migr. 27: 1-6.

- Grémillet, D., Boulinier, T. 2009. Spatial ecology and conservation of seabirds facing global climate change: a review. Mar. Ecol. Progr. Ser. 391: 121-137.

- INCC 2013. Seabird population trends and causes of change: 1996-2012. Report. Joint Nature Conservation Comittee.

- Juez, L., Aldalur. A., Herrero, A., Galarza, A. Arizaga, J. 2015. Effect of age, colony of origin and year on survival of a resident yellow-legged gull Larus michahellis population in the Bay of Biscay. Ardeola 62(1).

- Mitchell, P. I., Newton, S.F., Ratcliffe, N., Dunn, T.E. 2006. Seabird populations of Britain and Ireland: the last 30 years. In: Waterbirds around the world. G.C. Boere, C.A. Galbraith, D.A. Stroud (Eds): 765-766. The Stationery Office. Edinburgh.

- Molina, B.E. 2009. Gaviota reidora, sombría y patiamarilla en España. Población en 2007-2009 y método de censo. SEO/BirdLife. Madrid.

- Newton, I. 2013. Bird Populations. HarperCollins Publishers. London.

- Olea, P.P., Baglioni, V. 2008. Population trends of Rooks Corvus frugilegus in Spain and the importante of refuse tips. Ibis 150: 98-109.

- Oro, D., Martínez-Abraín, A. 2007. Decostructing myths on large gulls and their impact on threatened sympatric waterbirds. Animal Conserv. 10: 117-126. 
- Oro, D., Genovart, M., Tavecchia, G., Fowler, M.S., Martínez-Abraín, A. 2013. Ecological and evolutionary implications of food subsidies from humans. Ecol. Lett. 16:1501-1514.

- PIGRUB, 2012. Segunda revisión ordinaria del Plan Integral de Gestión de Residuos Urbanos de Bizkaia 2005-2016. CIMAS.

- Pons, J.M. 1992. Effects of changes in the availability of human refuse on breeding parameters in a Herring Gull Larus argentatus population in Brittany, France. Ardea 80: 143-150.

- Pons, J.M., Migot, P. 1995. Life-History Strategy of the Herring Gull: Changes in Survival and Fecundity in a Population Subjected to Various Feeding Conditions. J. Animal Ecol. 64: 592-599.

- Raven, S.J., Coulson, J.C. 1997. The distribution and abundance of large gulls nesting in buildings in Britain and Ireland. Bird Study 44: 13-34.

- Saurola, P. 2009. Bad news and good news: population changes of Finnish owls during 19822007. Ardea 97: 469-482

\section{岁}

- Fecha de recepción/Date of reception: 14/10/2014

- Fecha de aceptación/Date of acceptance: 12/02/2015

Editor Asociado/Associate editor: David Álvarez 